\title{
Lansoprazole inhibits mitochondrial superoxide production and cellular lipid peroxidation induced by indomethacin in RGM1 cells
}

\author{
Kanho Rai, ${ }^{1}$ Hirofumi Matsui, ${ }^{1, *}$ Tsuyoshi Kaneko, ${ }^{1}$ Yumiko Nagano, ${ }^{1}$ Osamu Shimokawa, ${ }^{1}$ Jumpei Udo, ${ }^{1}$ \\ Aki Hirayama, ${ }^{2}$ Ichinosuke Hyodo, ${ }^{1}$ Hiroko P. Indo ${ }^{3}$ and Hideyuki J. Majima ${ }^{3}$ \\ 'Graduate School of Comprehensive Human Sciences, University of Tsukuba, 1-1-1 Tennodai, Tsukuba, Ibaraki 305-8575, Japan \\ ${ }^{2}$ The Center of Integrative Medicine, Tsukuba University of Technology, 4-12-7 Kasuga, Tsukuba, Ibaraki 305-8575, Japan \\ ${ }^{3}$ The Department of Oncology and Department of Space Environmental Medicine, Kagoshima University Graduate School of Medical and Dental Sciences, \\ 8-35-1 Sakuragaoka, Kagoshima 890-8544, Japan
}

(Received 10 October, 2010; Accepted 22 October, 2010; Published online 17 June, 2011)

\begin{abstract}
Lansoprazole is effective in healing non-steroidal anti-inflammatory drugs induced ulcers, and antioxidant properties have been thought to play a key role in healing ulcers. We hypothesize that lansoprazole exerts a cytoprotective effect by inhibiting reactive oxygen species leakage from mitochondria and lipid peroxidation. We pretreated gastric epithelial RGM1 cells with lansoprazole and then treated them with indomethacin in vitro. We found that the lansoprazole pretreatment significantly reduced cellular injury, maintained mitochondrial transmembrane potential, and decreased lipid peroxidation. Furthermore, the signal intensity of the electron spin resonance spectrum of the indomethacin-treated mitochondria which were pretreated with lansoprazole showed considerable reduction compared to those without the lansoprazole pretreatment. These results suggest that lansoprazole reduced superoxide production in the mitochondria of indomethacin treated cells, and subsequently inhibited lipid peroxide and cellular injury in gastric epithelial cells.
\end{abstract}

Key Words: lansoprazole, superoxide, lipid peroxidation, electron spin resonance, indomethacin

ansoprazole belongs to the class of proton pump inhibitors (PPIs) which are effective in healing non-steroidal antiinflammatory drugs (NSAIDs)-induced ulcers and preventing their recurrence in patients requiring ongoing NSAIDs therapy. ${ }^{(1,2)}$

Lansoprazole has been thought to prevent NSAIDs-induced ulcers and cellular injury through an effect that inhibits acid secretion, but this is not the only important effect. A meta-analysis showed that $\mathrm{H}_{2}$-receptor antagonists $\left(\mathrm{H}_{2} \mathrm{RAs}\right)$ which also inhibited acid secretion did not significantly reduce the risk of symptomatic ulcers among patients receiving NSAIDs. ${ }^{(3)}$

The difference between PPIs and $\mathrm{H}_{2}$ RAs can be attributed to the strength of acid suppression, while the cytoprotective effects of lansoprazole have been reported, as well as acid secretion inhibitory effects. ${ }^{(4,5)}$

NSAIDs-induced gastric injuries have been reported to be derived by their pharmacological effects by inhibiting cyclooxygenase (COX) from depleting mucosal prostaglandin E2 (PGE2) concentration. Lansoprazole has not been reported to influence PGE2 mucosal concentration. ${ }^{(6)}$ Therefore, the cytoprotective effect should be derived from the other physiological mechanisms without depletion of PGE2.

There were several reports that NSAIDs treatment involved both the uncoupling of mitochondrial oxidative phosphorylations and the inhibition of the electron transport chain with various kinds of cells or isolated mitochondria. ${ }^{(7)}$ This results in depletion of intracellular ATP and the generation of reactive oxygen species (ROS). ${ }^{(8-10)}$ ROS may lead to lipid peroxidation and apoptosis by releasing cytochrome $\mathrm{c}^{(11,12)}$

We hypothesize that lansoprazole exerts a cytoprotective effect by inhibiting ROS leakage from mitochondria and lipid peroxidation. Lansoprazole has been reported to decrease the tissue oxidative stress induced by NSAIDs treatment. ${ }^{(4,13)}$ The protective effects of lansoprazole against NSAIDs-induced oxidative stresses may be derived from a direct reaction which decreases mitochondrial ROS production. However, there has been no report to confirm which kind of ROS can be involved after the NSAIDs treatment, or to confirm the details of lansoprazole's pharmacological effects for NSAIDs-induced tissue oxidative stresses.

In this study, we elucidated which kind of ROS was generated and whether or not lansoprazole showed a direct protective effect for ROS generation after indomethacin, a NSAID treatment. We examined both indomethacin-induced ROS production and cellular tissue peroxidation with and without lansoprazole pretreatment using our established normal gastric epithelial cell-line, RGM1. In particular, we confirmed the kind of ROS produced from indomethacin-treated cellular mitochondria with both an electron spin resonance (ESR) technique and a new spin trapping reagent 5(2,2-Dimethyl-1,3-propoxy cyclophosphoryl)-5-methyl-1-pyrroline $\mathrm{N}$-oxide (CYPMPO). Using this technique, we also examined the effects of lansoprazole on mitochondrial ROS generation after the indomethacin treatment.

\section{Materials and Methods}

Cell culture. The previously established gastric epithelial cell line, RGM1, was reobtained by our laboratory from RIKEN BioResource Center (Tsukuba, Japan). Cells were grown in a $1: 1$ mixture of both Dulbecco's modified Eagle medium and Ham's F-12 medium (DMEM/F12; Cosmo Bio, Tokyo, Japan) supplemented with $10 \%$ fetal calf serum (FCS; Gibco, Grand Island, NY) and $2 \mathrm{mM}$ glutamine at $37^{\circ} \mathrm{C}$ in a humidified incubator with $5 \%$ $\mathrm{CO}_{2}$.

Reagents. Indomethacin was purchased from WAKO Co. (Osaka, Japan), Lansoprazole was obtained from Takeda Pharmaceutical Co. (Osaka, Japan), prostaglandin E2 was purchased from Cayman Chemical Co., Ltd. (Ann Arbor, Michigan), DMEM/F12

*To whom correspondence should be addressed. E-mail: hmatsui@md.tsukuba.ac.jp 
was purchased from Cosmo Bio Co., Ltd., FCS was purchased from Gibco BRL, Tetra Color ONE ${ }^{\circledR}$ cell proliferation assay kit from Seikagaku (Tokyo, Japan), Diphenyl-1pyrenylphosphine (DPPP) and 5,5',6,6'-tetrachloro-1,1',3,3'-tetraethylbenzimidazolylcarbocyanine iodide (JC-1) from Dojindo (Kumamoto, Japan), 5(2,2-Dimethyl-1,3-propoxy cyclophosphoryl)-5-methyl-1-pyrroline N-oxide (CYPMPO) from Radical Research Inc. (Tokyo, Japan). All other chemicals were reagent grade.

MnSOD c-DNA transfection. The pCR3.1-Uni plasmid (Invitrogen, Carlsbad, CA) containing a sense human MnSOD (manganese superoxide dismutase) cDNA insert was kindly supplied by Dr. Akashi (National Institute of Radiological Sciences, Chiba, Japan). Sequence analysis of the MnSOD gene in the construct showed that the sequence was identical to that of the gene with GenBank Accession No. Y00472, except that C at position 113 was changed to $\mathrm{T}$, and $\mathrm{C}$ at position 529 was changed to $\mathrm{G}$, which caused changes from alanine to valine and glutamine to glutamic acid, respectively. The RGM1 cells were transfected using the Lipofectamine ${ }^{\mathrm{TM}} 2000$ reagent transfection system (Invitrogen) in accordance with the manufacturer's instructions. Briefly, cells were plated onto 60-mm dishes and incubated for $24 \mathrm{~h}$ before transfection at $80 \%$ confluence. The cells were stably transfected with $5 \mu \mathrm{g}$ of pCR3.1-Uni plasmids containing a sense human MnSOD cDNA insert, and linearized by Sca I, in a serumfree DMEM/F12, and five hours after transfection, the medium was changed to DMEM/F12 containing $10 \%$ fetal bovine serum. The controls were transfected with pCR3.1-Uni plasmids without a human MnSOD cDNA (vector alone) insert and linearized by Sca I. Stable clones of both MnSOD and control plasmid transfectants were selected with Geneticin (Life Technologies, Carlsbad, $\mathrm{CA})$ at a final concentration of $500 \mu \mathrm{g} / \mathrm{ml}$. Selected cellular clones that expressed MnSOD (MnSOD-4, -6 and -8), selectable marker alone (vector), and the parental cell (RGM1) were used in all of the experiments. Selected clones were routinely maintained in DMEM containing 10\% fetal bovine serum (JRH Biosciences, Lenexa, KS) and $500 \mu \mathrm{g} / \mathrm{ml}$ Geneticin at $37^{\circ} \mathrm{C}$ in humidified air containing $5 \% \mathrm{CO}_{2}$.

Cytotoxicity assay. Cellular injury was examined with the Tetra Color One assay kit according to the manufacturer's instructions. Cells at a concentration of $10^{5}$ cells $/ \mathrm{ml}$ were placed on a microtiter plate well. Twenty-four hours later, cells were washed with phosphate buffered saline (PBS; Gibco) and pretreated with lansoprazole, omeprazole, and control media for $24 \mathrm{~h}$, respectively, then washed with PBS and treated with indomethacin. After $18 \mathrm{~h}$ incubation, then washed with PBS and treated with serum-free media containing $10 \mu \mathrm{l}$ Tetra Color One for $1 \mathrm{~h}$. The absorbance of each well at $450 \mathrm{~nm}$ was measured with a multimode plate reader (DTX 880; Beckman Coulter, Fullerton, CA).

Cellular injury was also examined by MnSOD-overexpressed cells (MnSOD-4, -6 and -8). Cells were pretreated with lansoprazole for $24 \mathrm{~h}$, then washed with PBS and treated with indomethacin. After $24 \mathrm{~h}$ incubation, they were washed with PBS and treated with serum-free media containing $10 \mu \mathrm{l}$ Tetra Color One for $1 \mathrm{~h}$. The absorbance of each well was measured with the same methods mentioned above.

Detection of lipid peroxidation. Diphenyl-1-pyrenylphosphine (DPPP) reacts specifically with hydroperoxides and hydrogen peroxide that are generated within cell membranes to give fluorescent diphenyl-1-pyrenylphosphine oxide (DPPP oxide), which emits fluorescence with excitation and emission wavelengths of 351 and $380 \mathrm{~nm}$, respectively. Cells incubated in medium on a slide chamber were pretreated with lansoprazole for $24 \mathrm{~h}$ and then treated with indomethacin for $18 \mathrm{~h}$. After washing with PBS and incubating with DMEM/F12 containing $10 \mu \mathrm{M}$ DPPP for $30 \mathrm{~min}$, cellular fluorescent images were observed and their intensities were measured with a chilled CCD camera (AxioCam color, ZEISS, Germany)-mounted epi-fluorescence microscope (Axiovert135M,
ZEISS) connected to an image analyzing system (Axio Vision, ZEISS).

Mitochondrial membrane potential detection. Mitochondrial membrane potential was measured with JC-1. In healthy cells, JC-1 accumulates as aggregates in the mitochondria which stain red, whereas, if the mitochondrial membrane potential collapses, JC-1 exists in monomeric form and stains the cytosol green. The aggregate red form has an absorption/emission maxima of $585 / 590 \mathrm{~nm}$. The green monomeric form has an absorption/ emission maxima of $510 / 527 \mathrm{~nm}$. Cells at a concentration of $10^{5}$ cells $/ \mathrm{ml}$ were placed on a microtiter plate well, and cells were pretreated with lansoprazole for $24 \mathrm{~h}$, then washed with PBS and treated with indomethacin. After $18 \mathrm{~h}$ incubation, cells were washed with PBS and incubated with serum-free media containing JC-1. After 15 min incubation, the cells were washed with PBS and analyzed by fluorescence plate reader (DTX-880; Bechman Coulter, Fullerton, CA).

Electron spin resonance spectroscopy. Reactive oxygen species were measured by ESR. Cells were pretreated with lansoprazole for $24 \mathrm{~h}$, then washed with PBS. Then, cells were treated with indomethacin for $4 \mathrm{~h}$. After administration of reagents, cells were gently scraped off the dish, and isolated to mitochondria. A Mitochondria Isolation Kit for Cultured cells (Pierce Biotechnology, Inc. Rockford, IL) was used for this procedure. The pellet of mitochondria was resuspended with $5 \mathrm{mM}$ respiratory substrates (succinate, glutamate and malate), $5 \mathrm{mM}$ NADH and $10 \mathrm{mM}$ CYPMPO. The reaction mixture was immediately transferred to a quartz flat cell (RDC-60;60 $\mathrm{mm} \times 6 \mathrm{~mm} \times 0.3 \mathrm{~mm}$, Radical Research). The concentration of proteins in the final reaction mixture was $250 \mu \mathrm{g} / \mathrm{ml}$ as evaluated according to the method described previously (Bio-Rad Laboratories, Hercules, CA). The ESR spectra were recorded by using a JEOL-TE X-band spectrometer (JEOL, Tokyo, Japan). All ESR spectra were obtained under the following conditions: $10 \mathrm{~mW}$ incident microwave power, $100 \mathrm{kHz}$ modulation frequency, $0.1 \mathrm{mT}$ field modulation amplitude and $15 \mathrm{mT}$ scan range. Spectral computer simulation were performed using a Win-Rad Radical Analyzer System (Radical Research). All ESR spectra shown are representative of at least three independent experiments.

Statistical analysis. The statistical significance of the data was evaluated using analysis of variance (ANOVA) followed by Duncan's multiple range test. Statistical comparisons were made using Scheffe's method. A $p$ value of $<0.05$ was considered significant.

\section{Results}

Effects of lansoprazole against indomethacin-induced gastric cell damage. To examine the protective effect of lansoprazole against indomethacin-induced cellular damage, we undertook a cell viability assay in gastric epitherial RGM-1 cells, which have no potential for acid secretion.

The exposure of RGM1 cells to $750 \mu \mathrm{M}$ indomethacin for $18 \mathrm{~h}$ caused a significant dose-related loss of cell viability, as determined by the Tetra-Color ONE method (Fig. 1). The concentration of indomethacin was decided according to previous preliminary studies. Pretreatment with lansoprazole for $24 \mathrm{~h}$ before the indomethacin treatment significantly prevented the viability loss caused by $750 \mu \mathrm{M}$ indomethacin treatment. These results indicated that lansoprazole has a protective effect against indomethacin-induced cellular damage regardless of any acid secretion inhibitory effect.

Evaluation of lipid peroxidation by DPPP method. Lipid peroxidation was involved in indomethacin-induced cellular injury. To examine whether lansoprazole pretreatment reduces indomethacin-induced lipid peroxidation in gastric epithelial cells, we pretreated RGM-1 cells with lansoprazole and examined lipid peroxidation by detecting a fluorescent maker DPPP-oxide, a specific marker of lipid peroxidation (Fig. 2). The fluorescence 


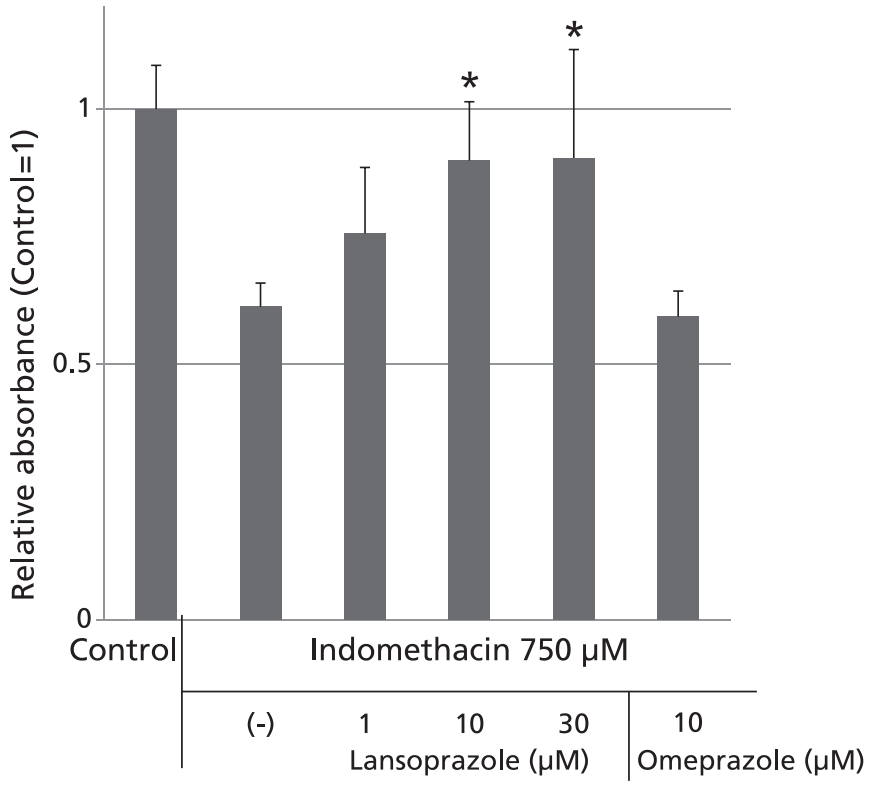

Fig. 1. Indomethacin induced cellular injury was measured by TetraColor One method. The pretreatment of lansoprazole reduced cytotoxicityin a dose-dependent $(1,10,30 \mu \mathrm{M})$ manner and absorbance of Tetra-Color One was significantly higher than those of the un-pretreated group. The viability losses induced by $750 \mu \mathrm{M}$ indomethacin were $38.7 \% \pm 4.6 \%$. Lansoprazole at 10 and $30 \mu \mathrm{M}$ significantly inhibited the reduction in cell viability, the inhibition being $14.2 \%$ at $10 \mu \mathrm{M}$ and $14.4 \%$ at $30 \mu \mathrm{M}$, respectively, as determined by the Tetra Color One assay method. On the other hand, the same amount of pretreatment of omeprazole had no cytoprotective effect in this study. ${ }^{*} p<0.05$ vs indomethacin $750 \mu \mathrm{M}$. intensity of DPPP-oxide was significantly increased in indomethacin-treated cells compared to that in control cells. The fluorescence intensity of DPPP oxide was significantly reduced in lansoprazole-pretreated cells compared to indomethacin-alonetreated cells, suggesting that lansoprazole pretreatment partly inhibited indomethacin-induced lipid peroxidation in RGM1 cells.

Effects of lansoprazole against indomethacin-induced disruption of mitochondrial membrane potential. Disruption of mitochondrial membrane potential is involved in lipid peroxidation induced by indomethacin treatment. ${ }^{(11)}$ We hypothesized that lansoprazole protected the disruption of mitochondrial membrane potential. We investigated mitochondrial transmembrane potentials of indomethacin-treated RGM1 cells pretreated with or without lansoprazole using a fluorescent indicator JC-1. The results clearly indicate that the fluorescent intensity significantly decreased in the indomethacin treated cells, and that the lansoprazole pretreatment significantly inhibited the decrease of the fluorescent intensity compared to that of indomethacin treated cells. These results suggest that lansoprazole protected against the disruption of mitochondrial membrane potentials induced by indomethacin treatment (Fig. 3).

Determination of reactive oxygen species by electron spin resonance. Disruption of mitochondrial membrane potential by indomethacin treatment is accompanied with the release of reactive oxygen species. We hypothesized indomethacin-induced superoxide $\left(\mathrm{O}_{2}{ }^{--}\right)$leakage from mitochondria, because superoxide is a major source of mitochondrial ROS leakage. To investigate whether indomethacin treatment induced superoxide leakage from mitochondria, we undertook ESR spectroscopy of isolated mitochondria from indomethacin-treated RGM-1 cells by using a spin-trapping reagent, CYPMPO. Furthermore, effects of lansoprazole pretreatment on superoxide leakage were investigated. The mitochondria isolated from indomethacin treated RGM-1 cells that were pretreated with or without lansoprazole were examined. Incubation with respiratory substrates, the isolated mitochondria showed ESR spectra of CYPMPO-OOH (Fig. 4). The ESR spectrum parameters obtained from the mitochondria from the cells treated with indomethacin alone were quite similar to those of produced by the hypoxanthine (HX)/xanthine oxidase (XOD)

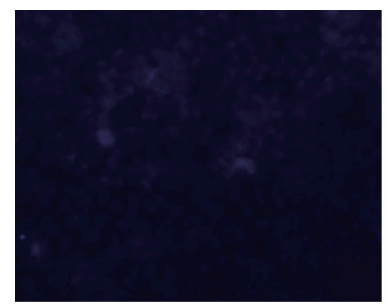

Control

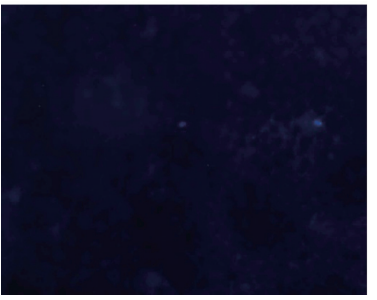

Lansoprazole

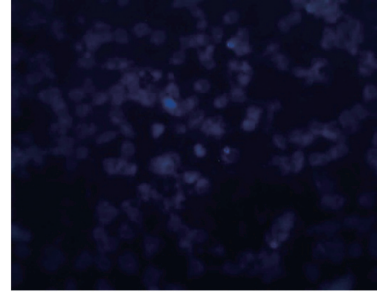

Lansoprazole (LPZ) + Indomethacin (IND)

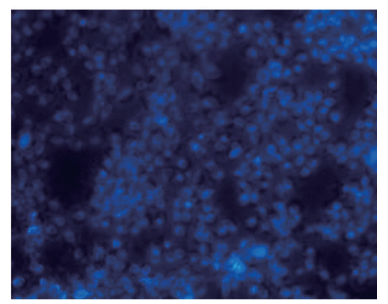

Indomethacin

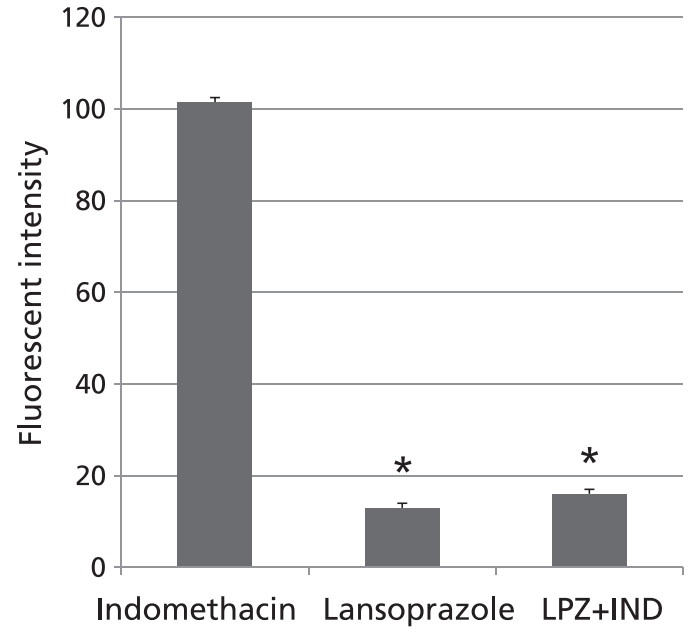

Fig. 2. The indomethacin-induced cellular lipid peroxidation was measured with DPPP-oxide fluorescence. Fluorescence intensity was measured with a high-sensitivity camera-mounted epifluorescence microscope system. Cells with the indomethacin treatment emitted detectable DPPP fluorescence, and $24 \mathrm{~h}$ pretreatment of lansoprazole significantly reduced the intensity of DPPP fluorescence $(101.5 \pm 8.9 \mathrm{vs} 16.0 \pm 4.0)$. ${ }^{*} p<0.05 \mathrm{vs}$ indomethacin. 
system and a simulation of CYPMPO-OOH adduct. The hyperfine stricture constants (hfsc) of the spectrum agreed with that of the computer-simulated spectrum of CYPMPO-OOH (data not shown). Therefore, we concluded that the spectrum was assigned to the CYPMPO-OOH spin adduct formed by the reaction of superoxide with CYPMPO. The signal intensity of this ESR spectrum of the mitochondria of the indomethacin-treated cells pretreated with lansoprazole was considerably reduced in comparison to that of

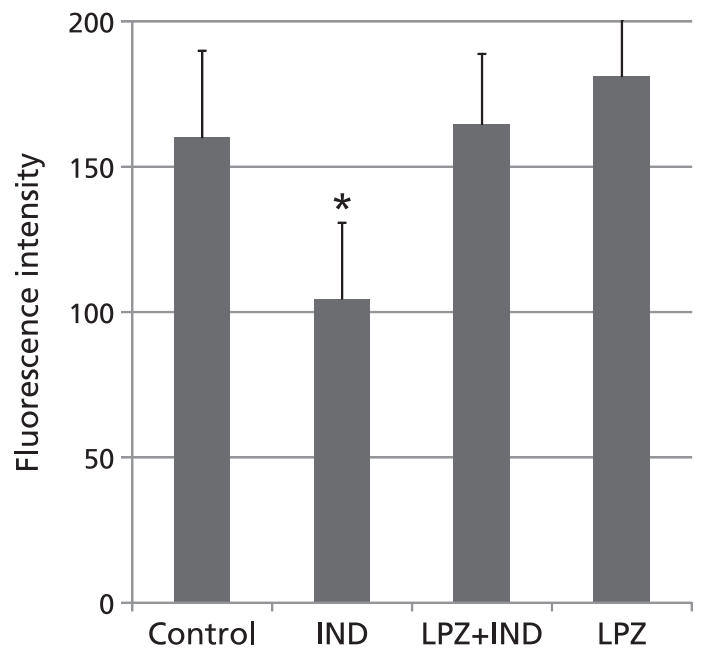

Fig. 3. Mitochondrial membrane potentials were investigated with JC1. Fluorescence intensity was measured with plate reader DTX 880 at $18 \mathrm{~h}$ of $750 \mu \mathrm{M}$ indomethacin treatments. The JC-1 fluorescence intensity of cells without indomethacin-treatment was significantly higher than that of indomethacin-treated cells. $24 \mathrm{~h}$ pretreatment of lansoprazole protected mitochondrial membrane potentials at almost normal levels $(160.0 \pm 29.0$ vs $104.5 \pm 26.3) .{ }^{*} p<0.05$ vs control. the cells which were not pretreated with lansoprazole. These results indicate that the mitochondria of the indomethacin-treated RGM1 cells produced superoxide, while the pretreatment with lansoprazole inhibited the production of superoxide.

Prevention of the indomethacin-induced cellular injury in MnSOD-overexpressed cells. The superoxide production has a key role in indomethacin-induced cellular damage, and previous results demonstrated that lansoprazole reduced the production of superoxide from mitochondria. We re-examined whether the inhibition of mitochondrial superoxide production is involved in the cytoprotective action of lansoprazole in indomethacin-induced cellular injury by treating the gastric cells that overexpressed MnSOD, which scavenges superoxide, with indomethacin and lansoprazole. The MnSOD stable clones (MnSOD4, MnSOD6 and MnSOD8) and vector alone clone (Vector) were constructed, and their activity was confirmed by native polyacrylamide gel electrophoresis SOD activity analysis. It is noted that the SOD activity of cytosolic copper zinc superoxide dismutase ( $\mathrm{Cu} / \mathrm{ZnSOD}$ ), which removes cytosolic superoxide, was not changed among the clones (data not shown).

RGM1 cells that overexpressed MnSOD or vector cells were pretreated with lansoprazole and treated with indomethacin and their cellular damage was examined with previously-mentioned methods. TC-1 analysis indicated that the cellular viability of indomethacin-treated RGM1 vector cells was significantly increased in the lansoprazole-pretreated group. On the other hand, there were no significant effects of treatment with vs without lansoprazole in MnSOD-overexpressed cells (Table 1). These results suggest that the inhibition of mitochondrial superoxide production is involved in the cytoprotective action of lansoprazole.

\section{Discussion}

In the presenting study we demonstrated that, for the first time to our knowledge, treatment of lansoprazole inhibits the leakage of superoxide from mitochondria, and subsequently protects against
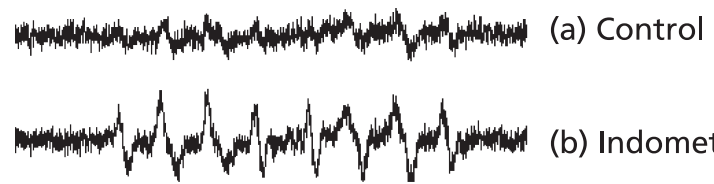

(b) Indomethacin

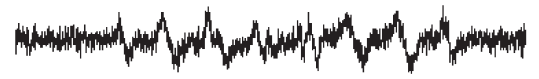

(c) Lansoprazole + Indomethacin

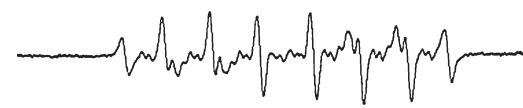

(d) HX/XOD

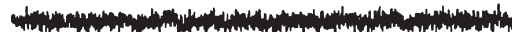

(e) MnSOD + indomethacin

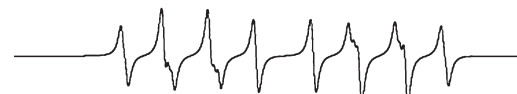

(f) Simulation

$335 \quad 340$

Field (mT)

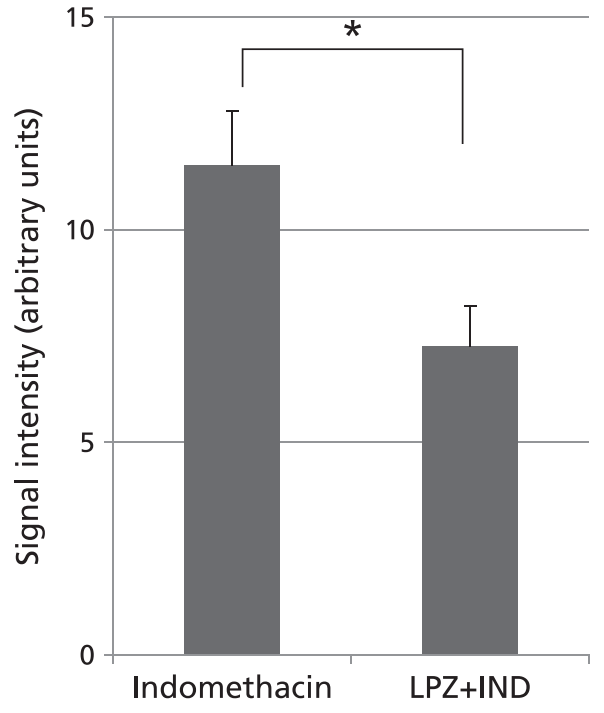

Fig. 4. ESR spectrum (a) was obtained when aqueous solution containing isolated mitochondria from RGM1 cells with respiratory substrates and $10 \mathrm{mM}$ CYPMPO was incubated for 5 min. ESR spectra (b) and (c) was obtained when aqueous solution containing indomethacin treated isolated mitochondria with (b) or without (c) lansoprazole pretreatment from RGM1 cells with respiratory substrates and 10 mM CYPMPO was incubated for $5 \mathrm{~min}$. ESR spectrum (d) was obtained when aqueous solution containing $250 \mu \mathrm{M}$ hypoxanthine, $25 \mathrm{mU} / \mathrm{ml}$ hypoxanthine oxidase, $0.1 \mathrm{mM}$ DATAPAC and $10 \mathrm{mM}$ CYPMPO was incubated for $5 \mathrm{~min}$. ESR spectrum (e) was obtained when aqueous solution containing indomethacin treated isolated mitochondria from MnSOD-overexpressed RGM1 cells and $10 \mathrm{mM}$ CYPMPO was incubated for 5 min. The ESR spectrum (f) was a computer simulated ESR spectrum. The amount of ROS leakage from the mitochondria without indomethacin-treated cells was significantly lower than that of indomethacin-treated cells. $24 \mathrm{~h}$ pretreatment of lansoprazole reduced this indomethacin-induced ROS leakage by half. ${ }^{*} p<0.05$. 
Table 1. Cell viability in manganese superoxide dismutase (MnSOD)overexpressed cells

Cell viability (\%)

\begin{tabular}{lcc}
\hline & Lansoprazole $(-)$ & Lansoprazole $(+)$ \\
\hline RGM1 & $26.5 \pm 0.2$ & $34.0 \pm 0.1^{*}$ \\
MnSOD4 & $64.5 \pm 0.2$ & $58.0 \pm 0.8$ \\
MnSOD6 & $60.1 \pm 0.5$ & $65.7 \pm 0.4$ \\
MnSOD8 & $56.0 \pm 0.3$ & $59.4 \pm 0.5$ \\
\hline
\end{tabular}

There were no significant effects of treatment with vs without lansoprazole in terms of cellular viability. mean $\pm S D,{ }^{*} p<0.05$ vs lansoprazole (-).

the cellular lipid peroxidation and cell death induced by indomethacin in vitro.

In past studies the effectiveness of lansoprazole has been examined by treating rodents with lansoprazole and NSAIDs in vivo. However we have recently demonstrated that gastric acid by itself induced the uncoupling of mitochondria, resulting in superoxide production and cellular lipid peroxidation. These results suggest that the effects of lansoprazole treatment in in vivo studies made it difficult to differentiate the effects due to acid suppression from those not caused by acid suppression. We established an in vitro study that was designed to allow the examination of the effects of lansoprazole beyond acid suppression: we used gastric epithelial RGM-1 cells that have no acid secretory ability and treated them with lansoprazole to measure mitochondria injury, superoxide generation, lipid peroxidation and cellular injury.

There is evidence that proton pump inhibitors, including lansoprazole, have not only acid-suppression effects but also antiinflammatory effects beyond acid suppression: the expression of $\mathrm{CD} 11 \mathrm{~b} / \mathrm{CD} 18$ at the neutrophil, the induction of heme oxygenase1 in the gastric epithelial cells, the suppression of IL-8 production in the gastric epithelial cells and the suppression of ICAM-1 and VCAM-1 in the inner cells. ${ }^{(14-16)}$ Moreover, it has been reported that proton pump inhibitors have a suppressive effect of reactive oxygen species in the neutrophil.(17) It was reported that omeprazole has a cytoprotective effect beyond acid suppression; however, the same dose of omeprazole was not able to protect against cellular injury in this study. The reason why the omeprazole was not able to protect against cellular injury by indomethacin is unknown, but there is a possibility that lansoprazole has a stronger cytoprotective effect than omeprazole. Indeed, an in vivo study recently demonstrated that lansoprazole, but not omeprazole, protected against an indomethacin-induced small intestine ulcer.

NSAIDs cause gastrointestinal injury through both topical and systemic effects. The systemic effects of NSAIDs have been well studied from the early 1970s, and the studies show that the reduction in prostaglandin biosynthesis is insufficient to explain fully the pathogenesis of NSAIDs-induced ulcers. ${ }^{(7)}$ Several studies show that reagents which have antioxidant potential were effective in preventing NSAIDs-induced ulcers. ${ }^{(18,19)}$ These results indicate that prostaglandin-independent mechanisms are important, and that, furthermore, decreasing ROS is effective in NSAIDsinduced ulcer. ROS probably directly oxidizes cellular proteins, lipids or nucleic acids and causes general damage or dysfunction, and probably initiates the cell death process through affecting various signaling cascades leading to necrosis and apoptosis. ${ }^{(20)}$ We elucidated that lansoprazole directly inhibits NSAIDs-induced ROS production and its derived cytotoxic reaction.

In the present study we did not show the effects of lansoprazole on PGE2 production. However, since treatment of PGE2 did not affect the injury of the mitochondria, we concluded the lansoprazole would not inhibit the pathway of the ROS generation from mitochondria by PGE2 production. In previous studies lansoprazole administration did not significantly modify prostaglandin release in gastric juice in rat models. ${ }^{(21,22)}$ Moreover, lansoprazole does not influence the down-regulation of gastric prostaglandin production associated with NSAID treatment. ${ }^{(6)}$ Therefore, lansoprazole does not appear to exert a positive influence on gastric PGE2 levels by NSAID treatment.

Gastric cellular lipid peroxidation has been regarded as an important prime event that leads to gastric mucosal injuries, and our previous study demonstrated that NSAIDs induced intrinsic, mitochondria-derived ROS, which evokes cellular peroxidation. Moreover, NSAIDs uncouple mitochondrial oxidative phosphorylation and inhibit the electron transport chain. This results in reduction of gastric mucosal ATP, induction of $\mathrm{Ca}^{2+}$ signaling and generation of ROS-like superoxide and hydroxyl radicals. ${ }^{(8-10)}$ However, it has been difficult to trap the superoxide stably. The formerly-used spin traps such as DMPO have a short half life for the superoxide adduct. CYPMPO can more efficiently trap superoxide compared with DMPO because it has lower cytotoxicity and its superoxide adduct has a longer lifetime. ${ }^{(23)}$ We trapped the superoxide from the indomethacin-treated mitochondria by using CYPMPO in ESR. These results were in agreement with our previous study that indomethacin causes lipid peroxidation by the superoxide from mitochondria.

In the present study, lansoprazole treatment reduced the superoxide from indomethacin-treated mitochondria. The exact mechanisms that caused lansoprazole to reduce the superoxide leakage from mitochondria remain to be fully elucidated; it seems probable that scavenging of the superoxide from indomethacin-treated mitochondria prevented lipid peroxidation. We also demonstrated that lansoprazole pretreated RGM1-vector cells were more efficient at protecting against indomethacin-induced cellular injury than MnSOD-overexpressed cells. These results suggest lansoprazole is not irrelevant to MnSOD.

In conclusion, this study demonstrated that lansoprazole reduced superoxide production in mitochondria of indomethacin treated cells, and subsequently inhibited lipid peroxide and cellular injury in gastric epithelial cells. The results provide biological evidence for effects beyond acid suppression of lansoprazole, and we expect that these findings will also be applicable in NSAIDs-induced small-intestinal ulcer which is independent from acid exposure.

\section{Acknowledgments}

We thank Ms. Marianne Kimura (Medical English Communication Center, University of Tsukuba, Japan) for critical review of the manuscript. We thank Professor Dr. Yashige Kotake (Oklahoma Medical Research Foundation, USA) for offering the simulation chart of ESR spectrum.

\section{Abbreviations}

\section{COX cyclooxygenase}

CYPMPO 5-(2,2-Dimethyl-1,3-propoxy cyclophosphoryl)-5methyl-1-pyrroline $\mathrm{N}$-oxide

JC-1 5,5',6,6'-tetrachloro-1,1',3,3'-tetraethylbenzimidazolylcarbocyanine iodide

$\mathrm{Cu} / \mathrm{ZnSOD}$ copper zinc superoxide dismutase

DPPP Diphenyl-1pyrenylphosphine

ESR electron spin resonance

$\mathrm{H}_{2} \mathrm{RAs} \quad \mathrm{H}_{2}$-receptor antagonists

Hfsc hyperfine stricture constants

HX hypoxanthine

MnSOD manganese superoxide dismutase

NSAIDs non-steroidal anti-inflammatory drugs

PBS phosphate buffered saline

PGE2 prostaglandin E2

PPIs proton pump inhibitors

ROS reactive oxygen species

XOD xanthine oxidase 


\section{References}

1 Lazzaroni M, Bianchi Porro. Prophylaxis and treatment of non-steroidal antiinflammatory drug-induced upper gastrointestinal side-effects. Dig Liver Dis 2001; 33 Suppl. 2: S44-S58

2 Singh G, Triadafilopoulos G. Appropriate choice of proton pump inhibitor therapy in the prevention and management of NSAID-related gastrointestinal damage. Int J Clin Pract 2005; 59: 1210-1217.

3 Hooper L, Brown TJ, Elliott R, Payne K, Roberts C, Symmons D. The effectiveness of five strategies for the prevention of gastrointestinal toxicity induced by non-steroidal anti-inflammatory drugs: systematic review. $B M J$ 2004; 329: 948.

4 Fornai M, Natale G, Colucci R, and et al. Mechanisms of protection by pantoprazole against NSAID-induced gastric mucosal damage. Naunyn Schmiedebergs Arch Pharmacol 2005; 372: 79-87.

5 Biswas K, Bandyopadhyay U, Chattopadhyay I, Varadaraj A, Ali E, Banerjee RK. A novel antioxidant and antiapoptotic role of omeprazole to block gastric ulcer through scavenging of hydroxyl radical. J Biol Chem 2003; 278: 10993-11001.

6 Blandizzi C, Fornai M, Colucci R, and et al. Lansoprazole prevents experimental gastric injury induced by non-steroidal anti-inflammatory drugs through a reduction of mucosal oxidative damage. World J Gastroenterol 2005; 11: 4052-4060.

7 Musumba C, Pritchard DM, Pirmohamed M. Review article: cellular and molecular mechanisms of NSAID-induced peptic ulcers. Aliment Pharmacol Ther 2009; 30: 517-531.

8 Tanaka $\mathrm{K}$, Tomisato $\mathrm{W}$, Hoshino $\mathrm{T}$, and et al. Involvement of intracellular $\mathrm{Ca}^{2+}$ levels in nonsteroidal anti-inflammatory drug-induced apoptosis. $J$ Biol Chem 2005; 280: 31059-31067.

9 Kusuhara H, Komatsu H, Sumichika H, Sugahara K. Reactive oxygen species are involved in the apoptosis induced by nonsteroidal anti-inflammatory drugs in cultured gastric cells. Eur J Pharmacol 1999; 383: 331-337.

10 Mahmud T, Rafi SS, Scott DL, Wrigglesworth JM, Bjarnason I. Nonsteroidal antiinflammatory drugs and uncoupling of mitochondrial oxidative phosphorylation. Arthritis Rheum 1996; 39: 1998-2003.

11 Nagano Y, Matsui H, Muramatsu M, and et al. Rebamipide significantly inhibits indomethacin-induced mitochondrial damage, lipid peroxidation, and apoptosis in gastric epithelial RGM-1 cells. Dig Dis Sci 2005; 50 Suppl. 1: S76-S83.

12 Yoshikawa T, Naito Y, Kishi A, and et al. Role of active oxygen, lipid peroxidation, and antioxidants in the pathogenesis of gastric mucosal injury induced by indomethacin in rats. Gut $1993 ; \mathbf{3 4 :} 732-737$.

13 Pastoris O, Verri M, Boschi F, and et al. Effects of esomeprazole on glutathione levels and mitochondrial oxidative phosphorylation in the gastric mucosa of rats treated with indomethacin. Naunyn Schmiedebergs Arch Pharmacol 2008; 378: 421-429.

14 Yoshida N, Yoshikawa T, Tanaka Y, and et al. A new mechanism for antiinflammatory actions of proton pump inhibitors-inhibitory effects on neutrophil-endothelial cell interactions. Aliment Pharmacol Ther 2000; 14 Suppl. 1: 74-81.

15 Handa O, Yoshida N, Fujita N, and et al. Molecular mechanisms involved in anti-inflammatory effects of proton pump inhibitors. Inflamm Res 2006; $\mathbf{5 5}$ 476-480.

16 Takagi T, Naito Y, Yoshikawa T. The expression of heme oxygenase-1 induced by lansoprazole. J Clin Biochem Nutr 2009; 45: 9-13.

17 Wandall JH. Effects of omeprazole on neutrophil chemotaxis, super oxide production, degranulation, and translocation of cytochrome b-245. Gut 1992; 33: 617-621.

18 Naito Y, Kajikawa H, Mizushima K, and et al. Rebamipide, a gastroprotective drug, inhibits indomethacin-induced apoptosis in cultured rat gastric mucosal cells: association with the inhibition of growth arrest and DNA damage-induced 45 alpha expression. Dig Dis Sci 2005; 50 Suppl. 1: S104-S112.

19 Sivalingam N, Hanumantharaya R, Faith M, Basivireddy J, Balasubramanian $\mathrm{KA}$, Jacob M. Curcumin reduces indomethacin-induced damage in the rat small intestine. $J$ Appl Toxicol 2007; 27: 551-560.

20 Orrenius S. Reactive oxygen species in mitochondria-mediated cell death Drug Metab Rev 2007; 39: 443-455.

21 Natale G, Lazzeri G, Lubrano V, and et al. Mechanisms of gastroprotection by lansoprazole pretreatment against experimentally induced injury in rats: role of mucosal oxidative damage and sulfhydryl compounds. Toxicol Appl Pharmacol 2004; 195: 62-72.

22 Fukuda T, Arakawa T, Shimizu Y, Ohtani K, Higuchi K, Kobayashi $\mathrm{K}$. Effects of lansoprazole on ethanol-induced injury and PG synthetic activity in rat gastric mucosa. J Clin Gastroenterol 1995; 20 Suppl. 2: S5-S7.

23 Kamibayashi M, Oowada S, Kameda H, and et al. Synthesis and characterization of a practically better DEPMPO-type spin trap, 5-(2,2-dimethyl-1,3propoxy cyclophosphoryl)-5-methyl-1-pyrroline N-oxide (CYPMPO). Free Radic Res 2006; 40: 1166-1172. 